\section{Arteria hepática izquierda de arteria gastroduodenal. Un desafío técnico durante cirugía de Whipple. Caso clínico}

\author{
RUBÉN ÁVILA S., GUILLERMO VANDER STELT A., \\ ALFONSO NAVIA T., ALEJANDRO ALTAMIRANO M., \\ JUAN FCO. GUERRA C., JORGE MARTÍNEZ C., NICOLÁS JARUFE C.
}

\section{Left hepatic artery (LHA) emerging from the gastroduodenal artery. Report of one case}

Anatomic variations of the hepatic artery, which occur in 30 to $50 \%$ of patients, are a very important factor to be considered for Whipple procedure. The most common variations are those coming from the superior mesenteric artery, left gastric artery and the aorta. We report a 58-year-old woman with a story of one month of epigastric pain, jaundice and progressive itching. Magnetic resonance imaging showed a mass in the head of the pancreas. During pancreatoduodenectomy a left hepatic artery (LHA) emerging from the gastroduodenal artery and an accessory LHA emerging from the left gastric artery, were observed. The rest of the surgery was performed with no incidents. The patient had an uneventful postoperative evolution.

(Rev Med Chile 2015; 143: 673-676)

Key words: Anatomic variation; Hepatic artery; Pancreatoduodenectomy; Surgical procedures, operative.

$\mathrm{P}$ ancreaticoduodenectomía es el único tratamiento curativo para el cáncer de la región periampular. Esta cirugía continúa siendo un procedimiento complejo de elevada morbilidad y mortalidad ${ }^{1-4}$, es por ello que la adecuada planificación preoperatoria así como la realización de esta cirugía por un equipo entrenado y en un centro de alto volumen, son recomendables ${ }^{5,6}$. Junto a lo anterior, las variaciones anatómicas de la arteria hepática son muy importantes de tener presentes en procedimientos quirúrgicos, tales como trasplante hepático, donante vivo, cirugía gástrica o pancreática, así como de radiología intervencional relacionada. El hígado tiene un suministro de sangre dual que se deriva de la arteria hepática propia $(30 \%)$ y de la vena porta $(70 \%)$, el patrón dominante que da origen a la arteria hepática propia desde la arteria hepática común originada en el tronco celiaco en $50-70 \%$

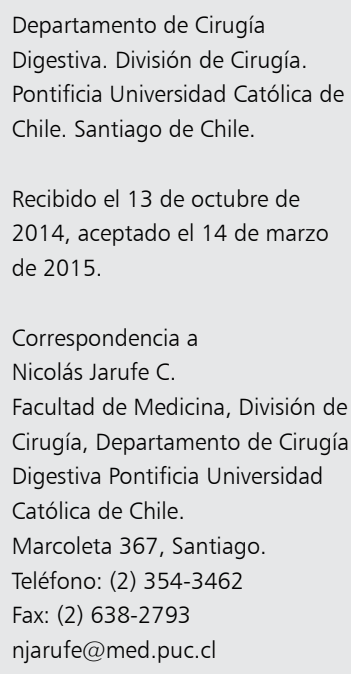

de los $\operatorname{casos}^{7}$. Existen variaciones del origen de las arterias hepáticas con una incidencia de alrededor de $30-50 \%$, siendo las más frecuentes aquellas que se originan de arteria mesentérica superior, gástrica izquierda y aorta. El desconocimiento y la sección inadvertida o errónea de estas arterias hepáticas variantes puede llevar a necrosis de uno o más segmentos hepáticos o a problemas biliares intrahepáticos que en ocasiones constituyen un desafío mayor en términos de tratamiento; ya que pueden requerir de múltiples intervenciones, resecciones hepáticas e incluso trasplante.

\section{Caso clínico}

Paciente de 58 años de edad, con antecedentes médicos de hipertensión arterial (HTA) y quirúrgicos de resección de condrosarcoma en peroné 
Tabla 1. Resultados de exámenes de laboratorio durante evolución post operatoria

\begin{tabular}{|lcccccc|}
\hline $\begin{array}{l}\text { Días post operatorio } \\
\text { Exámenes de lab. }\end{array}$ & $\begin{array}{c}\mathbf{6}^{\circ} \\
\text { Post op }\end{array}$ & $\begin{array}{c}\mathbf{1 0}^{\circ} \\
\text { Post op }\end{array}$ & $\begin{array}{c}\mathbf{1 1}^{\mathbf{0}} \\
\text { Post op }\end{array}$ & $\begin{array}{c}\mathbf{1 5}^{\circ} \\
\text { Post op }\end{array}$ & $\begin{array}{c}\mathbf{2 0}^{\circ} \\
\text { Post op }\end{array}$ & $\begin{array}{c}\mathbf{2 9}^{\circ} \\
\text { Post op }\end{array}$ \\
\hline Hematocrito (\%) & 33 & 30 & 29 & 30 & 33 & 39 \\
\hline Leucocitos/mm & 10.700 & 23.000 & 17.300 & 11.000 & 7.200 & 11.600 \\
\hline PCR (mg/dl) & & 15,9 & 12,11 & 3,8 & 0,75 & 1,38 \\
\hline Bilirrubina Total (mg/dl) & 10,9 & 6,16 & 5,6 & 3,3 & 2,25 & 1,9 \\
\hline Bilirrubina directa (mg/dl) & 9,00 & 5,29 & 4,6 & 2,9 & 2,05 & 1,7 \\
\hline Fosfatasa alcalina (U/L) & 522 & 1115 & 1408 & 1184 & 764 & 578 \\
\hline GGT (U/L) & 378 & 805 & 910 & 869 & 731 & 713 \\
\hline GOT (U/L) & 59 & 56 & 68 & 49 & 36 & 35 \\
\hline GPT (U/L) & 166 & 87 & 86 & 54 & 28 & 26 \\
\hline
\end{tabular}

PCR: proteína C reactiva; GGT: gamma glutamil transpeptidasa; GOT: transaminasa glutámico-oxaloacética; GPT: transaminasa glutámico-pirúvica.

izquierdo distal y halux valgus. Con historia de 1 mes de molestias epigástricas inespecíficas asociadas a saciedad precoz, ictericia, coluria y prurito nocturno, baja de peso de $5 \mathrm{~kg}$ en este período. Pruebas hepáticas destaca bilirrubina total: 16,3 $\mathrm{mg} / \mathrm{dl}$, bilirrubina directa: $14 \mathrm{mg} / \mathrm{dl}$, fosfatasas alcalinas (FA): $827 \mathrm{U} / \mathrm{L}$, gamma glutamiltranspeptidasa (GGT): $698 \mathrm{U} / \mathrm{L}$, transaminasa glutámico-oxalácetico (GOT): $170 \mathrm{U} / \mathrm{L}$, transaminasa glutámico-pirúvica (GPT): $426 \mathrm{U} / \mathrm{L}$. Hematocrito: $35,1 \%$ leucocitos: $5.250 / \mathrm{mm}^{3}$, plaquetas 34.100 / $\mathrm{mm}^{3}$, Ca 19-9: 1.240. Resonancia nuclear magnética de abdomen mostraba masa de cabeza pancreática de $47 \mathrm{~mm}$ de aspecto anterior, que estenosaba conducto colédoco intrapancreático proximal, provocando marcada dilatación de la vía biliar hasta $22 \mathrm{~mm}$ de diámetro en el hepático común. No se observaba coledocolitiasis. Sin diseminación intraabdominal evidente. Tomografía computada (TAC) tórax sin evidencias de diseminación torácica. Se realizó cirugía de Whipple el 07 de junio de 2014 (pancreatoduodenectomía sin preservación pilórica), durante la resección del tumor se observó variación anatómica, donde la arteria hepática izquierda emergía de la arteria gastroduodenal (AGD) vecina a la masa pancreática (Figura 1). Se avanzó en disección del pedículo identificando la arteria hepática derecha proveniente de arteria hepática común, distal a emergencia de gastroduodenal. No se logró identificar otra arteria desde la hepática común aparte de la arteria hepática derecha. Se observó la hepática izquierda accesoria proveniente de la gástrica izquierda que irrigaba los segmentos laterales (II y III). Dada la necesidad de seccionar la arteria gastroduodenal en su origen por la cercanía con el tumor, se decidió sección de arteria gástrica izquierda proveniente de la AGD apreciando en ese momento flujo arterial retrógrado que significaba irrigación de ese territorio probablemente por accesoria de la gástrica izquierda y algunas comunicantes de la derecha. Dado este hallazgo, se decidió no reconstruir la arteria hepática izquierda y mantener el flujo arterial hepático por la derecha más la accesoria de la gástrica izquierda. Se continuó resección de tumor sin incidentes. Se realizó reconstrucción con pancreato-yeyuno anastomosis ducto-mucosa sin stent. El paciente evolucionó en buenas condiciones en post operatorio, con fistula pancreática tipo $\mathrm{A}^{8}$, de bajo débito. Durante el décimo día post operatorio el paciente presentó fiebre asociada a aumento de parámetros inflamatorios y aumento de FA y GGT (Tabla 1). Se realizó TAC TAP que informó extensa área de infarto en los segmentos laterales del lóbulo hepático izquierdo (Figura 2). Evolucionó en forma favorable al tratamiento antibiótico con normalización de parámetros inflamatorios. Se retiró drenaje el día 21 postoperatorio previo confirmación de amilasas normales y fue dada de alta en buenas condiciones al día 23. El plan es controlar con imágenes la evolución de su infarto hepático segmentario. Biopsia diferida 


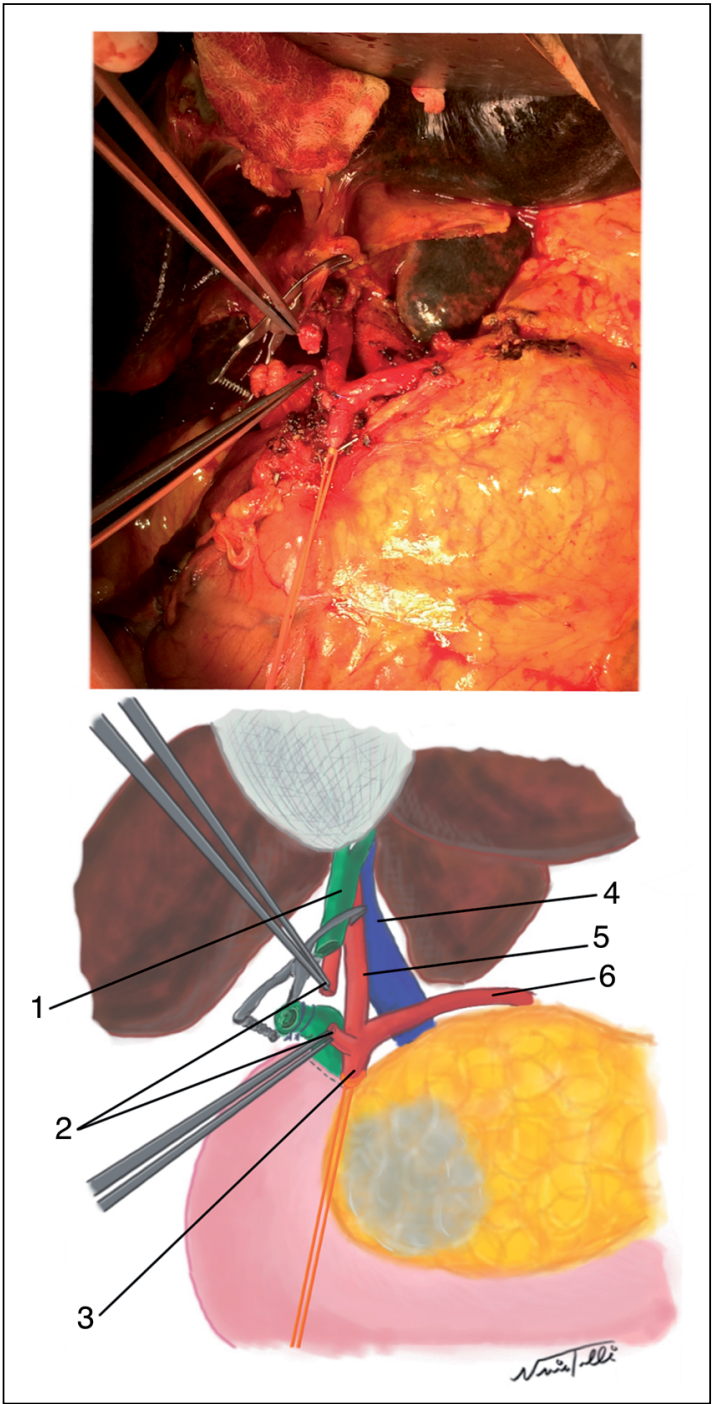

Figura 1. Fotografía intraoperatoria de cirugía de Whipple y su representación esquemática mostrando hallazgo de arteria hepática izquierda reemplazada desde arteria gastroduodenal. (1) Vía biliar seccionada; (2) arteria hepática izquierda reemplazada seccionada entre pinzas; (3) arteria gastroduodenal; (4) vena porta; (5) arteria hepática derecha; (6) arteria hepática común.

mostró: Cáncer de cabeza de páncreas nodular, tipo histológico adenocarcinoma ductal poco diferenciado infiltrante en pared duodenal, colédoco distal intrapancreático y tejido fibroconectivo peri-pancreático. Metástasis ganglionares linfáticas en 1 de 24 ganglios examinados. Borde quirúrgico del páncreas negativo. AJCC: pTM T3N1Mx.

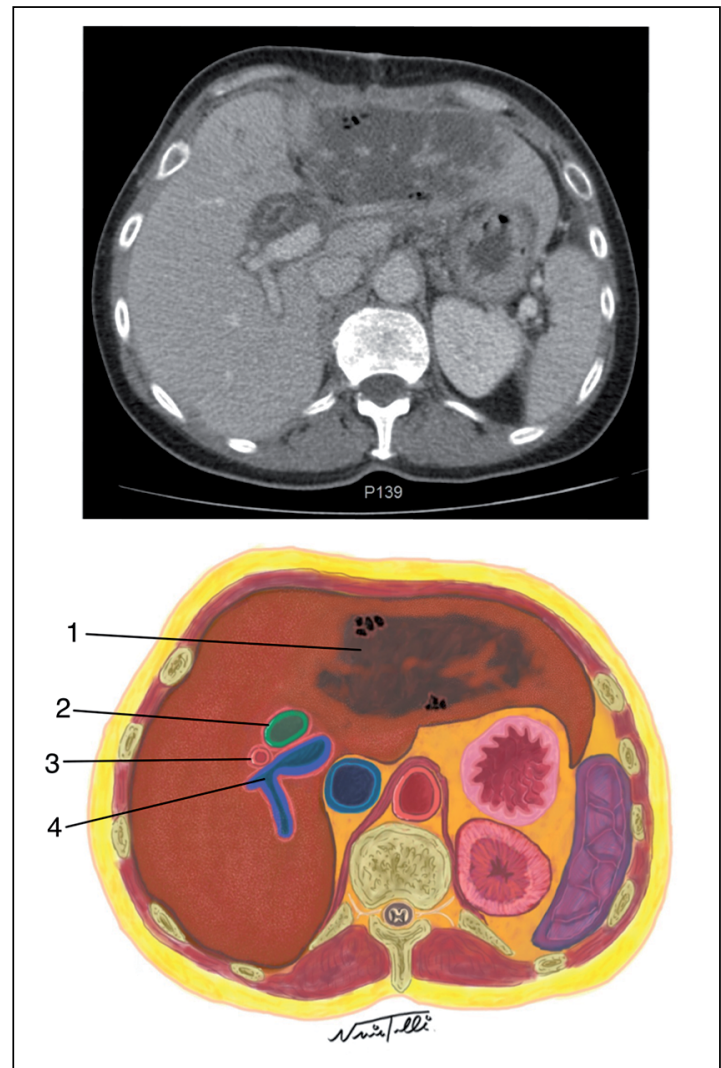

Figura 2. Corte transversal de TAC de abdomen con contraste y su representación esquemática mostrando necrosis de lóbulo hepático izquierdo. (1) Área de necrosis; (2) anastomosis biliodigestiva; (3) arteria hepática derecha; (4) vena porta ramificándose.

\section{Discusión}

Las variantes anatómicas de la arteria hepática tienen numerosas clasificaciones que se han realizado en base a autopsias (Michels 1966) ${ }^{9}$, radiología (Koop 2004) ${ }^{10}$ y trasplante (Hiatt $1994)^{11}$, siendo la descrita de excepción. Del total de variantes anatómicas vasculares de la irrigación arterial hepática (30-50\%), 16\% corresponde a variaciones en el origen de la arteria hepática común, $80 \%$ corresponde a variantes del origen de las arterias hepáticas derecha o izquierda y $4 \%$ corresponde a variantes raras ${ }^{7}$.

Se debe intentar preservar los vasos de distribución anatómica inhabitual. Dada la elevada incidencia de variaciones anatómicas de la irrigación arterial hepática, nos parece razonable más que 
plantear el estudio de angiografía de rutina, estar alerta en toda cirugía hepato-biliar y pancreática a estas variantes y saber reconocerlas para evitar daños que pueden traer serias consecuencias.

En el caso descrito, dada la presencia de una arteria hepática accesoria de la gástrica izquierda, se decide no re-anastomosar la arteria hepática izquierda con lo que además se elevaba el riesgo de dañar la arteria hepática derecha. La evolución de la paciente fue favorable, sin embargo, igualmente hubo daño biliar intrahepático de parte de los segmentos laterales a pesar de la arteria accesoria y del flujo arterial retrógrado obtenido al seccionar la arteria hepática izquierda. Por ahora no ha sido necesario ninguna intervención sobre este infarto pero en ocasiones es necesario resecar las zonas infartadas ya que pueden producir complicaciones biliares intrahepáticas (hepatolitiasis, abscesos).

\section{Referencias}

1. Shrikhande SV, Barreto G, Shukla PJ. Pancreatic fistula after pancreaticoduodenectomy: the impact of a standardized tech- nique of pancreaticojejunostomy. Langenbecks Arch Surg 2008; 393: 87-91.

2. Shukla PJ, Barreto SG, Mohandas KM, Shrikhande SV. Defining the role of surgery for complications after pancreatoduodenectomy. ANZ J Surg 2009; 79: 33-7.

3. Barreto SG, D'Souza MA, Shukla PJ, Shrikhande SV. The gray zone between post-pancreaticoduodenectomy collections and pancreatic fistula. Pancreas 2008; 37: 422-5.

4. Yeo CJ, Cameron JL, Sohn TA, Lillemoe KD, Pitt HA, Talamini MA, et al. Six hundred fifty consecutive pancreaticoduodenectomies in the 1990s: pathology, complications, and outcomes. Ann Surg 1997; 226: 248-57.

5. Ho V, Heslin MJ. Effect of hospital volumen and experience on in hospital mortality for pancreaticoduodenectomy. Ann Surg 2003; 237: 509-14.

6. Birkmeyer JD, Siewers AE, Finlayson EV, Stukel TA, Lucas FL, Batista I. Hospital Volume and surgical mortality in the united states. N Engl J Med 2002; 346: 1128-37.

7. Abdullah SS, Jean-Yves M, Garbit V, De La Roche E, Olagne E, Rode A, et al. Anatomical variations of the hepatic artery: study of 932 cases in liver transplantation. Surg Radiol Anat 2006; 28: 468-73.

8. Bassi C, Dervenis C, Butturini G, Fingerhut A, Yeo C, Izbicki J, et al. Postoperative pancreatic fistula: an international study group (ISGPF) definition. Surgery 2005; 138: 8-13.

9. Michels NA. Newer anatomy of the liver and its variant blood supply and collateral circulation. Am J Surg 1996; 112: 337-47.

10. Koops A, Wojciechowski B, Broering DC, Adam G, Krupski-Berdien G. Anatomic variations of the hepatic arteries in 604 selective celiac and superior mesenteric angiographies. Surg Radiol Anat 2004; 26 (3): 239-44.

11. Hiatt JR, Gabbay J, Busuttil RW. Surgical anatomy of the hepatic arteries in 1000 cases. Ann Surg 1994; 220: 50-2. 